\title{
Resonance fluorescence in a waveguide geometry
}

\author{
Şükrü Ekin Kocabaş, ${ }^{1, *}$ Eden Rephaeli, ${ }^{2, \dagger}$ and Shanhui Fan ${ }^{3, \ddagger}$ \\ ${ }^{1}$ Department of Electrical \& Electronics Engineering, Koç University, Rumeli Feneri Yolu TR-34450 Sartyer, İstanbul, Turkey \\ ${ }^{2}$ Department of Applied Physics, Stanford University, Stanford, California 94305, USA \\ ${ }^{3}$ Ginzton Laboratory, Department of Electrical Engineering, Stanford University, Stanford, California 94305, USA
}

(Received 1 December 2011; published 15 February 2012)

\begin{abstract}
We show how to calculate the first- and second-order statistics of the scattered fields for an arbitrary intensity coherent-state light field interacting with a two-level system in a waveguide geometry. Specifically, we calculate the resonance fluorescence from the qubit, using input-output formalism. We derive the transmission and reflection coefficients, and illustrate the bunching and antibunching of light that is scattered in the forward and backward directions, respectively. Our results agree with previous calculations on one- and two-photon scattering as well as those that are based on the master equation approach.
\end{abstract}

DOI: $10.1103 /$ PhysRevA.85.023817

PACS number(s): 42.50.Ct, 42.50.Ar, 42.50.Dv

\section{INTRODUCTION}

Interaction between an atom and a laser beam tuned close to one of the atomic resonances leads to light emission from the atom (i.e., resonance fluorescence) with a rich set of spectral and temporal properties. Spectrally, as the laser intensity is increased the emitted light will develop symmetric side lobes around the central excitation frequency and the resulting spectral shape is called the Mollow triplet [1]. Temporally, the light emitted will also show antibunching with a second-order correlation that has a minimum for zero time delay [2].

Recent advances in integrated optics [3,4] and superconducting circuits [5] make it possible to think about quantum systems connected to each other via waveguides that operate at optical or microwave frequencies. For such waveguide embedded systems, the Mollow triplet was observed in the emission spectra from a single superconducting qubit [6] and correlation measurements were also reported [7-9]. These structures were later shown to work as a switch [10] or a router [11]. In the optical domain, resonance fluorescence was modeled in photonic band-gap waveguides [12] and experimentally investigated in a system where a fiber was coupled to a quantum dot $[13,14]$.

Conventional modeling of resonance fluorescence focuses on light that is emitted in a direction perpendicular to the direction of the laser excitation [15-18] which results in antibunched statistics. In multiqubit systems it is possible to observe both bunching and antibunching due to the interference of light emission from different qubits [19-21]. In a waveguide geometry excitation and observation directions are colinear as shown in Fig. 1. The transmitted amplitudes have contributions from both the incident waves and the emitted waves from the atom. The resonance fluorescence effect is therefore different from the conventional situation. In a previous study based on two-photon scattering off of a qubit embedded in a waveguide, bunching and antibunching of light due to the interference of the incoming light with the scattered fields in the transmitted and reflected directions,

\footnotetext{
*ekocabas@ku.edu.tr

†edenr@stanford.edu

${ }^{\ddagger}$ shanhui@stanford.edu
}

respectively, was predicted [22]. In this work, as an original contribution, we will extend the two-photon analysis to the case where the excitation is made with an arbitrary intensity coherent state. We will make use of input-output formalism [23] recently generalized to waveguide structures [24] to derive analytical expressions for the second-order correlation functions for the reflected and transmitted fields. We will further show that the low excitation limit of the coherent-state solutions agrees very well with the two-photon results. The Mollow triplet will naturally emerge in our analysis. A distinct feature of our analysis is that we can calculate the multitime correlations without specifically referring to the quantum regression theorem.

The outline of this article is as follows. In Sec. II we will provide the necessary definitions and derive the single and double time correlations for one-way waveguides by using input-output formalism. In Sec. III we will extend the analysis to two-way waveguides and derive the spectra of the transmitted and reflected fields. Section IV will have the analysis on double time correlations for the scattered fields where we compare the coherent-state and the two-photon results. We will conclude the article in Sec. V.

\section{DERIVATION OF THE SINGLE AND DOUBLE TIME CORRELATIONS}

A system consisting of a qubit interacting with photons in a waveguide is described by the Hamiltonian $H=H_{0}+H_{1}$, where [24]

$$
\begin{aligned}
& H_{0}=\int_{-\infty}^{\infty} d \omega \omega a_{\omega}^{\dagger} a_{\omega}, \\
& H_{1}=\frac{1}{2} \Omega \sigma_{z}+\frac{V}{\sqrt{v_{g}}} \int_{-\infty}^{\infty} d \omega\left(\sigma_{+} a_{\omega}+a_{\omega}^{\dagger} \sigma_{-}\right) .
\end{aligned}
$$

Here $\omega$ is the atomic transition frequency, $a_{\omega}^{\dagger}$ and $a_{\omega}$ are the creation and annihilation operators for photons at frequency $\omega$, respectively. $\sigma_{-}$and $\sigma_{+}$are the lowering and raising operators for the qubit $\sigma_{z}=\left[\sigma_{+}, \sigma_{-}\right] . V$ denotes the coupling strength between the atomic states and the waveguide modes, and $v_{g}$ is the group velocity of the propagating waveguide mode. In the derivation of the Hamiltonian we make the dipole and 


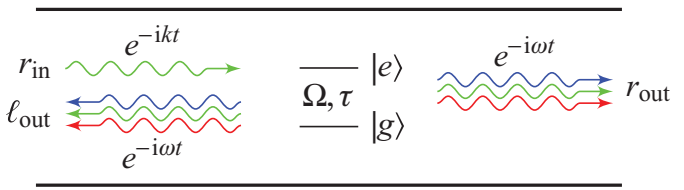

FIG. 1. (Color online) Schematic of the system under investigation. A right-going coherent state $\left(r_{\text {in }}\right)$ at frequency $k$ and an arbitrary intensity, propagating in a waveguide denoted by the long horizontal lines, is incident on a two-level system with energy separation $\Omega$ and a spontaneous emission rate $\tau^{-1}$. After interacting with the qubit, the transmitted $\left(r_{\text {out }}\right)$ and the reflected $\left(\ell_{\text {out }}\right)$ light has both a coherent $(\omega=k)$ and an incoherent $(\omega \neq k)$ component.

the rotating wave approximations, linearize the waveguide dispersion around the excitation frequency to obtain the group velocity, and assume that the photons are at a frequency in the vicinity of the excitation wavelength so that the linearization can be justified [24].

We set

$$
\begin{aligned}
\frac{1}{\tau} & =\pi \frac{V^{2}}{v_{g}}, \quad a_{\mathrm{in}}(t)=\frac{1}{\sqrt{2 \pi}} \int d \omega a_{\omega}\left(t_{0}\right) e^{-i \omega\left(t-t_{0}\right)}, \\
a_{\mathrm{out}}(t) & =\frac{1}{\sqrt{2 \pi}} \int d \omega a_{\omega}\left(t_{1}\right) e^{-i \omega\left(t-t_{1}\right)}
\end{aligned}
$$

where $a_{\text {in }}$ and $a_{\text {out }}$ are the input and output fields defined long before $\left(t_{0} \rightarrow-\infty\right)$ and long after $\left(t_{1} \rightarrow \infty\right)$ the interaction between the qubit and the the photons takes place. The two fields are related by

$$
a_{\mathrm{out}}(t)=a_{\mathrm{in}}(t)-i \sqrt{\frac{2}{\tau}} \sigma_{-}(t) .
$$

Through the help of the Heisenberg equations of motion and the definitions of the input and output fields, we can write the following set of input-output equations for a single qubit system [24]

$$
\begin{aligned}
& \frac{d \sigma_{-}(t)}{d t}=i \sqrt{\frac{2}{\tau}} \sigma_{z}(t) a_{\mathrm{in}}(t)-\left(\frac{1}{\tau}+i \Omega\right) \sigma_{-}(t), \\
& \frac{d \sigma_{+}(t)}{d t}=-i \sqrt{\frac{2}{\tau}} a_{\mathrm{in}}^{\dagger}(t) \sigma_{z}(t)-\left(\frac{1}{\tau}-i \Omega\right) \sigma_{+}(t),
\end{aligned}
$$

$$
\begin{aligned}
\frac{d \sigma_{z}(t)}{d t}= & -i 2 \sqrt{\frac{2}{\tau}}\left[\sigma_{+}(t) a_{\mathrm{in}}(t)-a_{\mathrm{in}}^{\dagger}(t) \sigma_{-}(t)\right] \\
& -\frac{2}{\tau}\left[\sigma_{z}(t)+1\right] .
\end{aligned}
$$

In this article we will be interested in the statistics of the scattered fields when a coherent-state input is incident on the qubit. We define the incoming coherent state at frequency $k$ as

$$
\left|\alpha_{k}^{+}\right\rangle=e^{-\left|\alpha_{k}\right|^{2} / 2} \sum_{n=0}^{\infty} \frac{\alpha_{k}^{n}}{\sqrt{n !}}\left|n_{k}^{+}\right\rangle=e^{-\left|\alpha_{k}\right|^{2} / 2} \sum_{n=0}^{\infty} \frac{\alpha_{k}^{n} a_{\text {in }}^{\dagger}(k)^{n}}{n !}|0\rangle
$$

such that

$$
\begin{aligned}
a_{\text {in }}(t)\left|\alpha_{k}^{+}\right\rangle & =\frac{1}{\sqrt{2 \pi}} \int d k^{\prime} a_{\text {in }}\left(k^{\prime}\right) e^{-i k^{\prime} t}\left|\alpha_{k}^{+}\right\rangle \\
& =\frac{\alpha_{k}}{\sqrt{2 \pi}} e^{-i k t}\left|\alpha_{k}^{+}\right\rangle=\frac{\omega_{\mathrm{R}}}{2} \sqrt{\frac{\tau}{2}} e^{i \phi-i k t}\left|\alpha_{k}^{+}\right\rangle .
\end{aligned}
$$

The value of $\alpha_{k}$ is in general complex valued. We define $\alpha_{k} \equiv$ $\left|\alpha_{k}\right| e^{i \phi} . \omega_{\mathrm{R}} \equiv 2\left|\alpha_{k}\right| / \sqrt{\pi \tau}$ is the Rabi frequency.

The expectation value of an operator $O$ is given as

$$
\langle O\rangle \equiv\left\langle\alpha_{k}^{+}|O| \alpha_{k}^{+}\right\rangle .
$$

To describe resonance fluorescence in a waveguide, three classes of correlation functions will be of importance: ones with one operator, ones with two operators at two different times, and ones with three operators at two different times, that is,

$$
\begin{aligned}
& \boldsymbol{c}_{1}\left(t=0, t^{\prime}\right)=\left(\begin{array}{c}
\left\langle\sigma_{-}\left(t^{\prime}\right)\right\rangle \\
\left\langle\sigma_{+}\left(t^{\prime}\right)\right\rangle \\
\left\langle\sigma_{z}\left(t^{\prime}\right)\right\rangle
\end{array}\right), \quad \boldsymbol{c}_{2}\left(t, t^{\prime}\right)=\left(\begin{array}{c}
\left\langle\sigma_{+}(t) \sigma_{-}\left(t^{\prime}\right)\right\rangle \\
\left\langle\sigma_{+}(t) \sigma_{+}\left(t^{\prime}\right)\right\rangle \\
\left\langle\sigma_{+}(t) \sigma_{z}\left(t^{\prime}\right)\right\rangle
\end{array}\right), \\
& \boldsymbol{c}_{3}\left(t, t^{\prime}\right)=\left(\begin{array}{c}
\left\langle\sigma_{+}(t) \sigma_{-}\left(t^{\prime}\right) \sigma_{-}(t)\right\rangle \\
\left\langle\sigma_{+}(t) \sigma_{+}\left(t^{\prime}\right) \sigma_{-}(t)\right\rangle \\
\left\langle\sigma_{+}(t) \sigma_{z}\left(t^{\prime}\right) \sigma_{-}(t)\right\rangle
\end{array}\right) .
\end{aligned}
$$

To calculate these expectation values, we use input-output equations (3) and multiply them from the left and the right with the necessary terms. ${ }^{1}$ We then take the expectation values, make use of Eq. (4) and the commutator $\left[a_{\text {in }}\left(t^{\prime}\right), \sigma_{-}(t)\right]=0$ for $t^{\prime} \geqslant t[25]$ to arrive at the following set of differential equations for all three classes of expectation values $(n=1,2,3)$

$$
\frac{d}{d t^{\prime}} \boldsymbol{c}_{n}\left(t, t^{\prime}\right)=\boldsymbol{B}\left(t^{\prime}\right) \boldsymbol{c}_{n}\left(t, t^{\prime}\right)+\boldsymbol{b}_{n}, \quad \text { where } \quad \boldsymbol{B}=\left(\begin{array}{ccc}
-(1 / \tau+i \Omega) & 0 & \frac{1}{2} i \omega_{\mathrm{R}} e^{-i k t^{\prime}} e^{i \phi} \\
0 & -(1 / \tau-i \Omega) & -\frac{1}{2} i \omega_{\mathrm{R}} e^{i k t^{\prime}} e^{-i \phi} \\
i \omega_{\mathrm{R}} e^{i k t^{\prime}} e^{-i \phi} & -i \omega_{\mathrm{R}} e^{-i k t^{\prime}} e^{i \phi} & -2 / \tau
\end{array}\right), \quad \boldsymbol{b}_{n}=\left(\begin{array}{c}
0 \\
0 \\
b_{n}
\end{array}\right)
$$

These are called the optical Bloch equations with radiative damping. For different $n$, the inhomogeneous term $b_{n}$ and the initial conditions at $t^{\prime}=t$ are different: $b_{1}=-\frac{2}{\tau}$,

\footnotetext{
${ }^{1}$ For instance, to get the second set, $c_{2}\left(t, t^{\prime}\right)$, we need to multiply (3) evaluated at time $t^{\prime}$ by $\sigma_{+}(t)$ from the left.
}

$b_{2}=-\frac{2}{\tau}\left\langle\sigma_{+}(t)\right\rangle$, and $b_{3}=-\frac{2}{\tau}\left\langle\sigma_{+}(t) \sigma_{-}(t)\right\rangle$. Previously, the same results were derived through the help of the quantum regression theorem $[16,18]$. However, the derivation here follows naturally within input-output formalism. In Appendix A we provide the derivation of the general solution to the Bloch equations and in Appendix B explicit solutions for all $\boldsymbol{c}_{n}$ are listed. 


\section{DERIVATION OF THE FLUORESCENCE SPECTRUM OF THE TRANSMITTED AND THE REFLECTED LIGHT}

Up till now, our analysis did not distinguish between rightand left-going waves. Indeed, the Hamiltonian we wrote was for a chiral (i.e., one-way) waveguide. For a regular two-way waveguide where fields propagate in both directions, the Hamiltonian has separate input and output operators for right $(r)$ and left $(\ell)$ propagating waves [24]. The equations of motion become

$$
\begin{aligned}
\frac{d \sigma_{-}}{d t}=i \sqrt{\frac{2}{\tau}} \sigma_{z} r_{\mathrm{in}}+i \sqrt{\frac{2}{\tau}} \sigma_{z} \ell_{\mathrm{in}}-\left(\frac{2}{\tau}+i \Omega\right) \sigma_{-}, \\
\frac{d \sigma_{+}}{d t}=-i \sqrt{\frac{2}{\tau}} r_{\mathrm{in}}^{\dagger} \sigma_{z}-i \sqrt{\frac{2}{\tau}} \ell_{\mathrm{in}}^{\dagger} \sigma_{z}-\left(\frac{2}{\tau}-i \Omega\right) \sigma_{+}, \\
\frac{d \sigma_{z}}{d t}=-i 2 \sqrt{\frac{2}{\tau}}\left[\sigma_{+}\left(r_{\mathrm{in}}+\ell_{\mathrm{in}}\right)-\left(r_{\mathrm{in}}^{\dagger}+\ell_{\mathrm{in}}^{\dagger}\right) \sigma_{-}\right] \\
-\frac{4}{\tau}\left[\sigma_{z}+1\right] .
\end{aligned}
$$

We can decompose the right and left input or output states as

$$
\begin{aligned}
& r_{\text {in/out }}(t)=\frac{a_{\text {in/out }}(t)+\stackrel{\circ}{\text { in/out }}_{\text {in }}(t)}{\sqrt{2}}, \\
& \ell_{\text {in/out }}(t)=\frac{a_{\text {in } / \text { out }}(t)-\stackrel{\circ}{\text { in/out }}_{1}(t)}{\sqrt{2}},
\end{aligned}
$$

and as a result arrive at the Hamiltonian $H=H_{0}+H_{1}$, where

$$
\begin{aligned}
& H_{0}=\int d \omega \omega\left(a_{\omega}^{\dagger} a_{\omega}+a_{\omega}^{\dagger} \stackrel{a}{\omega}_{\omega}\right), \\
& H_{1}=\frac{1}{2} \Omega \sigma_{z}+\frac{\sqrt{2} V}{\sqrt{v_{g}}} \int d \omega\left(\sigma_{+} a_{\omega}+a_{\omega}^{\dagger} \sigma_{-}\right) .
\end{aligned}
$$

The fields $a$ and $a$ are even and odd combinations, respectively, of the right and left propagating fields. The interacting part of the Hamiltonian $H_{1}$ depends on $a$ only and the $a$ dependence is solely in the noninteracting part $H_{0}$. Except for an additional term in $H_{0}{ }^{2}$ the two-way Hamiltonian is very similar to the chiral Hamiltonian in Eq. (1). Hence we will be able to make use of the results of the previous section in the analysis of two-way waveguides. To do so, we decompose a right-going coherent state with frequency $k$ into two separate (even and odd) channels [26]

$$
\begin{aligned}
& \exp \left[\alpha r_{\text {in }}^{\dagger}(k)-\alpha^{*} r_{\text {in }}(k)\right]|0\rangle \\
& =\exp \left[\alpha \frac{a_{\text {in }}^{\dagger}(k)+a_{\text {in }}^{\dagger}(k)}{\sqrt{2}}-\alpha^{*} \frac{a_{\text {in }}(k)+\stackrel{\circ}{\text { in }}(k)}{\sqrt{2}}\right]|0\rangle \\
& \equiv\left|\frac{\alpha_{k}^{+}}{\sqrt{2}} ; \frac{\alpha_{k}^{+}}{\sqrt{2}}\right\rangle
\end{aligned}
$$

(where the first term in the bra-ket refers to the even channel and the second term to the odd channel) such that

$$
a_{\text {in }}(t)\left|\frac{\alpha_{k}^{+}}{\sqrt{2}} ; \frac{\alpha_{k}^{+}}{\sqrt{2}}\right\rangle=\stackrel{\circ}{\text { in }}_{(}(t)\left|\frac{\alpha_{k}^{+}}{\sqrt{2}} ; \frac{\alpha_{k}^{+}}{\sqrt{2}}\right\rangle=\stackrel{\circ}{\text { out }}_{\text {(t) }}\left|\frac{\alpha_{k}^{+}}{\sqrt{2}} ; \frac{\alpha_{k}^{+}}{\sqrt{2}}\right\rangle
$$

\footnotetext{
${ }^{2}$ Note also that the extra factor of $\sqrt{2}$ in front of $V$ in $H_{1}$ will lead to a redefinition $\tau \rightarrow \tau^{\prime} \equiv \tau / 2$.
}

$$
\begin{aligned}
& =\frac{\alpha_{k}}{\sqrt{2} \sqrt{2 \pi}} e^{-i k t}\left|\frac{\alpha_{k}^{+}}{\sqrt{2}} ; \frac{\alpha_{k}^{+}}{\sqrt{2}}\right| \\
& =\frac{\omega_{\mathrm{R}}}{2} \sqrt{\frac{\tau^{\prime}}{2}} e^{i \phi-i k t}\left|\frac{\alpha_{k}^{+}}{\sqrt{2}} ; \frac{\alpha_{k}^{+}}{\sqrt{2}}\right|,
\end{aligned}
$$

where $\tau^{\prime} \equiv \tau / 2$ absorbs the $\sqrt{2}$ factor. As one can see, the odd channel is interaction-free and thus is an eigenstate of $\stackrel{\circ}{\text { in }}_{\text {in }}(t)=$ $\stackrel{\circ}{\text { out }}(t)$ whereas the even channel is subject to $H_{1}$. Nevertheless, it is the combination of both the even and odd channels that lead to the right- and left-going fields. The two-channel expectation value of an operator $O$ is defined as

$$
\left\langle\langle O\rangle \equiv\left\langle\frac{\alpha_{k}^{+}}{\sqrt{2}} ; \frac{\alpha_{k}^{+}}{\sqrt{2}}|O| \frac{\alpha_{k}^{+}}{\sqrt{2}} ; \frac{\alpha_{k}^{+}}{\sqrt{2}}\right\rangle .\right.
$$

To calculate the spectral properties of the transmitted fields, we need to calculate the Fourier transform of $\left\langle\left\langle r_{\text {out }}^{\dagger}(t) r_{\text {out }}(t+\right.\right.$ $\delta t)\rangle\rangle$ with respect to $\delta t$ (see Fig. 1). By using (7) we can write

$$
\begin{aligned}
& \left\langle\left\langle r_{\text {out }}^{\dagger}(t) r_{\text {out }}(t+\delta t)\right\rangle\right\rangle \\
& \quad=\frac{1}{2}\left\langle\left\langle\left[a_{\text {out }}^{\dagger}(t)+a_{\text {out }}^{\dagger}(t)\right]\left[a_{\text {out }}(t+\delta t)+a_{\text {out }}(t+\delta t)\right]\right\rangle .\right.
\end{aligned}
$$

The application of Eq. (2) with $\tau \rightarrow \tau^{\prime}$ results in

$$
\begin{aligned}
= & \frac{1}{2}\left[\omega_{\mathrm{R}}^{2} \frac{\tau^{\prime}}{2} e^{-i k \delta t}-i \omega_{\mathrm{R}} e^{-i \phi+i k t}\left\langle\left\langle\sigma_{-}(t+\delta t\rangle\right\rangle\right.\right. \\
& \left.+i \omega_{\mathrm{R}} e^{i \phi-i k(t+\delta t)}\left\langle\left\langle\sigma_{+}(t)\right\rangle\right\rangle+\frac{2}{\tau^{\prime}}\left\langle\left\langle\sigma_{+}(t) \sigma_{-}(t+\delta t)\right\rangle\right\rangle\right] .
\end{aligned}
$$

One can show that the two-channel expectation values of operators are the same as their single-channel expectation values [i.e., those in Eq. (5)] except for the substitution $\tau \rightarrow \tau^{\prime}$. The derivation can be made by using (6), taking the relative expectation values, and using (8) to simplify the results. Therefore, we can use the steady-state values from Appendix B to arrive at

$$
\begin{aligned}
\left\langle\left\langle r_{\text {out }}^{\dagger}(t) r_{\text {out }}(t+\delta t)\right\rangle\right\rangle= & \frac{1}{\tau^{\prime}} \frac{R^{2}}{4}\left(\frac{-1+D^{2}+\frac{1}{2} R^{2}}{1+D^{2}+\frac{1}{2} R^{2}}\right) e^{-i k \delta t} \\
& +\frac{1}{\tau^{\prime}}\left\langle\left\langle\sigma_{+}(t) \sigma_{-}(t+\delta t)\right\rangle\right\rangle,
\end{aligned}
$$

where

$$
D=(\Omega-k) \tau^{\prime}, \quad \text { and } \quad R=\omega_{\mathrm{R}} \tau^{\prime}
$$

To calculate the Fourier transform of this expression, we need to know $\left\langle\left\langle\sigma_{+}(t) \sigma_{-}(t+\delta t)\right\rangle\right\rangle$ for $\delta t<0$ as well. By using the identity $\left\langle\left\langle\sigma_{+}(t+\delta t) \sigma_{-}(t)\right\rangle\right\rangle=\left\langle\left\langle\sigma_{+}(t) \sigma_{-}(t+\delta t)\right\rangle\right\rangle^{*}$ we can see that the expectation values for $\delta t<0$ are related to those with $\delta t>0$ by complex conjugation. The Laplace transform results in Appendix B thus allow us to calculate the Fourier transform 
as

$$
\begin{aligned}
& G_{r}^{(1)}(\omega) \equiv \mathcal{F}_{\delta t}\left[\left\langle\left\langle r_{\text {out }}^{\dagger}(t) r_{\text {out }}(t+\delta t)\right\rangle\right]\right] \\
& \quad=\frac{1}{\tau^{\prime}} \frac{1}{\sqrt{2 \pi}} \frac{\frac{1}{2} R^{2}}{1+D^{2}+\frac{1}{2} R^{2}}\left[\pi \delta(\omega-k)\left(D^{2}+\frac{1}{2} R^{2} \frac{D^{2}+\frac{1}{2} R^{2}}{1+D^{2}+\frac{1}{2} R^{2}}\right)+\frac{R^{2}}{\tau^{\prime 5}} \frac{(\omega-k)^{2} \tau^{\prime 2}+4+\frac{1}{2} R^{2}}{|P[-i(\omega-k)]|^{2}}\right]
\end{aligned}
$$

where the function $P$ is as defined in Eq. (B4). We will use the noninteracting case, that is,

$$
\left.G_{r_{0}}^{(1)} \equiv \mathcal{F}_{\delta t}\left[\left\langle r_{\text {in }}^{\dagger}(t) r_{\text {in }}(t+\delta t)\right\rangle\right\rangle\right]=\frac{\sqrt{2 \pi} R^{2}}{4 \tau^{\prime}} \delta(\omega-k)
$$

for normalization. As a result, the coherent part of the correlation function, one which is proportional to $\delta(\omega-k)$, will be given by

$$
g_{r_{\mathrm{coh}}}^{(1)}=\frac{1}{1+D^{2}+\frac{1}{2} R^{2}}\left(D^{2}+\frac{1}{2} R^{2} \frac{D^{2}+\frac{1}{2} R^{2}}{1+D^{2}+\frac{1}{2} R^{2}}\right) .
$$

For reflected fields we need to do a similar analysis for $\left\langle\left\langle\ell_{\text {out }}^{\dagger}(t) \ell_{\text {out }}(t+\delta t)\right\rangle\right.$. By using (7) and (2) we can see that

$$
\left\langle\left\langle\ell_{\text {out }}^{\dagger}(t) \ell_{\text {out }}(t+\delta t)\right\rangle\right\rangle=\frac{1}{\tau^{\prime}}\left\langle\left\langle\sigma_{+}(t) \sigma_{-}(t+\delta t)\right\rangle\right\rangle .
$$

The Fourier transform of this term is given by

$$
\begin{aligned}
G_{\ell}^{(1)}(\omega) \equiv & \mathcal{F}_{\delta t}\left[\left\langle\left\langle\ell_{\text {out }}^{\dagger}(t) \ell_{\text {out }}(t+\delta t)\right\rangle\right]\right] \\
= & \frac{1}{\tau^{\prime}} \frac{1}{\sqrt{2 \pi}} \frac{\frac{1}{2} R^{2}}{1+D^{2}+\frac{1}{2} R^{2}} \\
& \times\left[\frac{1+D^{2}}{1+D^{2}+\frac{1}{2} R^{2}} \pi \delta(\omega-k)\right. \\
& \left.+\frac{R^{2}}{\tau^{\prime 5}} \frac{(\omega-k)^{2} \tau^{\prime 2}+4+\frac{1}{2} R^{2}}{|P[-i(\omega-k)]|^{2}}\right] .
\end{aligned}
$$

We again normalize with respect to the noninteracting case, and obtain

$$
g_{\ell_{\mathrm{coh}}}^{(1)}=\frac{1+D^{2}}{\left(1+D^{2}+\frac{1}{2} R^{2}\right)^{2}}
$$

for the coherently backscattered fields. The incoherent parts of the reflected and transmitted fields are equal to each other and are given by

$$
g_{\text {incoh }}^{(1)}=\frac{1}{\pi} \frac{1}{1+D^{2}+\frac{1}{2} R^{2}} \frac{R^{2}}{\tau^{\prime 5}} \frac{(\omega-k)^{2} \tau^{\prime 2}+4+\frac{1}{2} R^{2}}{|P[-i(\omega-k)]|^{2}} .
$$

In Fig. 2 spectral features of the transmitted and reflected fields are plotted. Note that the results (9) and (10) agree with those in Refs. [6,11].

\section{DERIVATION OF THE SECOND-ORDER CORRELATION FUNCTION OF THE TRANSMITTED AND REFLECTED LIGHT}

Now that we have calculated various first-order correlations and investigated spectral properties of scattered fields, we can start to look into the time-dependent statistics of the transmitted and reflected fields. To do so, we will begin by investigating the second-order correlation function in a chiral waveguide $g_{c}^{(2)}$ given by

$$
g_{c}^{(2)}(\delta t)=\lim _{t \rightarrow \infty} \frac{\left\langle a_{\mathrm{out}}^{\dagger}(t) a_{\mathrm{out}}^{\dagger}(t+\delta t) a_{\mathrm{out}}(t+\delta t) a_{\mathrm{out}}(t)\right\rangle}{\left\langle a_{\mathrm{out}}^{\dagger}(t) a_{\mathrm{out}}(t)\right\rangle\left\langle a_{\mathrm{out}}^{\dagger}(t+\delta t) a_{\mathrm{out}}(t+\delta t)\right\rangle} .
$$

By using (2) and the results from Appendix B it can be shown that

$$
\left\langle a_{\text {out }}^{\dagger}(t) a_{\text {out }}(t)\right\rangle=\frac{1}{\tau} \frac{R^{2}}{8}
$$

Similarly, after some algebra we arrive at the following formula for the Laplace transform of $g_{c}^{(2)}$

$$
\mathcal{L}_{\delta t}\left[g_{c}^{(2)}(\delta t)\right]=\frac{1}{s}+\frac{8}{1+D^{2}+\frac{1}{2} R^{2}} \frac{s\left(s+\frac{1}{\tau}\right)}{P(s)} .
$$

Using the expression above, we can show that

$$
\begin{aligned}
& \lim _{R \rightarrow 0} g_{c}^{(2)}(\delta t)=\frac{\psi_{k, p}^{(2)}(t, t+\delta t)}{\frac{1}{\sqrt{2}}\left[\psi_{k}^{(1)}(t) \psi_{p}^{(1)}(t+\delta t)+\psi_{p}^{(1)}(t) \psi_{k}^{(1)}(t+\delta t)\right]} \\
& =\left|1+\frac{4}{(D+i)^{2}} e^{-i \frac{|\delta t|}{\tau}(D-i)}\right|^{2} \text {, }
\end{aligned}
$$

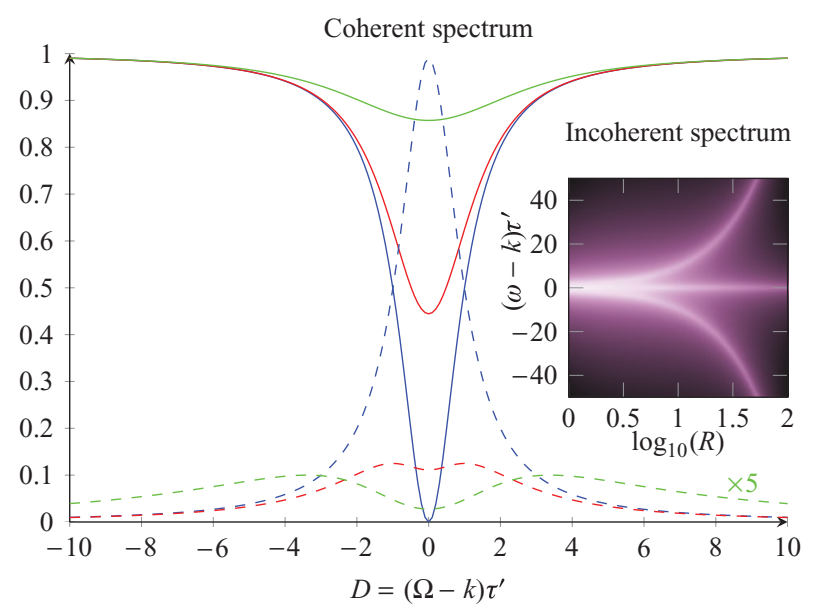

FIG. 2. (Color online) Coherent part of the transmitted (solid) and reflected (dashed) fluorescence spectrum for $R=\omega_{\mathrm{R}} \tau^{\prime}=\{0.1,2,5\}$ corresponding to the blue, red, and green curves respectively. The reflected fluorescence for $R=5$ (dashed green curve) is plotted after being multiplied by 5 . Inset shows the incoherent part ( $\omega \neq k$ case as depicted in Fig. 1) of the spectrum with the Mollow triplet for zero detuning $(D=0)$. 
(a)

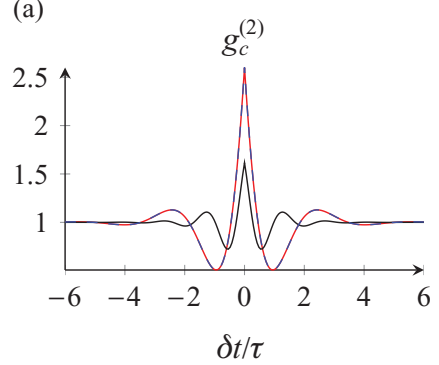

(b)

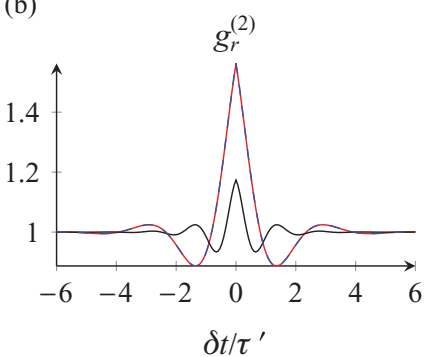

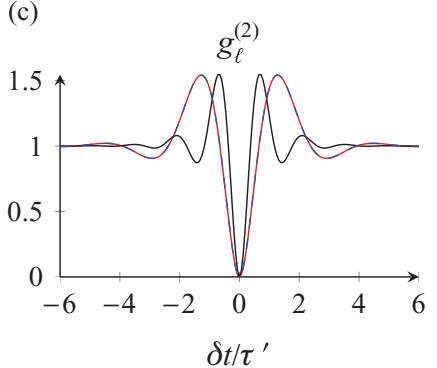

FIG. 3. (Color online) Plots of $g^{(2)}$ for $D=2$ and $R=\{0.2,4\}$ for the red and black curves, respectively. The dashed blue curve is the normalized two-photon wave function. (a) One-mode case; (b) two-mode, transmitted case; (c) two-mode, reflected case. As can be seen, the two-photon calculations are indistinguishable from resonance fluorescence ones for $R=0.2$ but not for $R=4$.

where $\psi^{(1)}\left[\psi^{(2)}\right]$ is the one-photon [two-photon] wave function. ${ }^{3}$ As a result, we have shown that the second-order statistics of a low-intensity coherent-state input and a twophoton input to a qubit are the same.

For the two-mode case, we will need to calculate the correlation functions for the right-going $(r)$ and the left-going ( $\ell$ ) fields as

$$
\begin{aligned}
& g_{r}^{(2)}(\delta t)=\lim _{t \rightarrow \infty} \frac{\left\langle\left\langle r_{\text {out }}^{\dagger}(t) r_{\text {out }}^{\dagger}(t+\delta t) r_{\text {out }}(t+\delta t) r_{\text {out }}(t)\right\rangle\right\rangle}{\left\langle\left\langle r_{\text {out }}^{\dagger}(t) r_{\text {out }}(t)\right\rangle\right\rangle\left\langle\left\langle r_{\text {out }}^{\dagger}(t+\delta t) r_{\text {out }}(t+\delta t)\right\rangle\right\rangle}, \\
& g_{\ell}^{(2)}(\delta t)=\lim _{t \rightarrow \infty} \frac{\left\langle\left\langle\ell_{\text {out }}^{\dagger}(t) \ell_{\text {out }}^{\dagger}(t+\delta t) \ell_{\text {out }}(t+\delta t) \ell_{\text {out }}(t)\right\rangle\right\rangle}{\left\langle\left\langle\ell_{\text {out }}^{\dagger}(t) \ell_{\text {out }}(t)\right\rangle\right\rangle\left\langle\left\langle\ell_{\text {out }}^{\dagger}(t+\delta t) \ell_{\text {out }}(t+\delta t)\right\rangle\right\rangle} .
\end{aligned}
$$

The normalization terms are given by

$$
\begin{aligned}
& \left\langle\left\langle r_{\text {out }}^{\dagger}(t) r_{\text {out }}(t)\right\rangle\right\rangle=\frac{1}{\tau^{\prime}} \frac{R^{2}}{4} \frac{D^{2}+\frac{1}{2} R^{2}}{1+D^{2}+\frac{1}{2} R^{2}}, \\
& \left\langle\left\langle\ell_{\text {out }}^{\dagger}(t) \ell_{\text {out }}(t)\right\rangle\right\rangle=\frac{1}{\tau^{\prime}} \frac{\frac{1}{4} R^{2}}{1+D^{2}+\frac{1}{2} R^{2}} .
\end{aligned}
$$

After some algebra done by the help of an automatic noncommutative algebra system [27] we get

$$
\begin{aligned}
\mathcal{L}_{\delta t} & {\left[g_{r}^{(2)}(\delta t)\right] } \\
= & \frac{1}{s}+\frac{1}{A^{2} P(s)} \\
& \times\left[\left(s+\frac{1}{\tau^{\prime}}\right)\left((1+2 A) s+\frac{1+4 A}{\tau^{\prime}}\right)+\frac{D^{2}}{\tau^{\prime 2}}\right],
\end{aligned}
$$

where $A=D^{2}+\frac{1}{2} R^{2}$, and

$$
\mathcal{L}_{\delta t}\left[g_{\ell}^{(2)}(\delta t)\right]=\frac{2}{\tau^{\prime 2}}\left(1+D^{2}+\frac{1}{2} R^{2}\right) \frac{s+\frac{1}{\tau^{\prime}}}{s P(s)} .
$$

In the limit $R \rightarrow 0$ the second-order correlation results for coherent-state and two-photon inputs in a two-mode waveguide can be shown to equal each other, just like in the chiral case (see Fig. 3). As was previously predicted, the interference of incoming and scattered fields leads to

\footnotetext{
${ }^{3}$ See equations (43) and (120) in Ref. [22] for the one- and twophoton wave functions, respectively. Note that the photons are at the same frequency (i.e., $k=p$ ).
}

bunching and antibunching in the forward and backward directions, respectively. When $R$ is increased, the response of the qubit gets saturated and there is less bunching in the forward direction but the reflected fields continue to show strong antibunching. In Refs. [28,29] $g^{(2)}$ was calculated for a low-intensity coherent-state interacting with a qubit in a waveguide where the qubit was coupled at a rate $\Gamma^{\prime}$ to nonwaveguide modes as well. Our results supplement these previous investigations by analytically describing the scattering of an arbitrary intensity ${ }^{4}$ coherent state off of a qubit for the $\Gamma^{\prime}=0$ case.

\section{CONCLUSION}

In this article we used input-output formalism for waveguides to analyze how an arbitrary intensity coherent state scatters off of a qubit embedded in a waveguide. We provided analytical results for the spectra as well as the second-order correlation functions of the transmitted and reflected fields. This work supplements the previous work on two-photon calculations and shows that the two formulations agree for low-intensity coherent-state inputs. We predicted that the transmitted fields are bunched and the reflected fields are antibunched for coherent-state inputs, similar to the case for two-photon scattering. Very recent experiments in circuit QED agree with these observations [30]. Functional devices (e.g., transistors [28], switches [10], or routers [11]) that make use of multilevel systems require both control signals that are in a coherent-state basis and single- or multi-photon Fock states that carry the information. We demonstrated the versatility of input-output formalism with which one can do analysis either based on Fock states to calculate the full scattering matrix, or based on coherent states with an emphasis on correlation measurements. Additionally, it is possible to investigate nonlinear effects such as the ac Stark [31] and Lamb [32] shifts using the methods developed to characterize qubit-coherent-state interactions. Lastly, our approach paves the way to calculations involving higher-order correlation functions that become relevant when the qubit is strongly excited; in a recent cavity QED experiment asymmetry in time for $g^{(3)}$ was demonstrated [33].

\footnotetext{
${ }^{4}$ We still operate within the bounds of the dipole and the rotatingwave approximations used in the derivation of the Hamiltonian.
} 


\section{ACKNOWLEDGMENTS}

This work is supported by the David and Lucile Packard Foundation.

\section{APPENDIX A: GENERAL SOLUTION TO THE BLOCH EQUATIONS}

In this Appendix we will provide the general solution to the differential equation

$$
\frac{d}{d t^{\prime}}\left(\begin{array}{l}
x_{1}\left(t, t^{\prime}\right) \\
x_{2}\left(t, t^{\prime}\right) \\
x_{3}\left(t, t^{\prime}\right)
\end{array}\right)=\boldsymbol{B}\left(t^{\prime}\right)\left(\begin{array}{c}
x_{1}\left(t, t^{\prime}\right) \\
x_{2}\left(t, t^{\prime}\right) \\
x_{3}\left(t, t^{\prime}\right)
\end{array}\right)+\left(\begin{array}{c}
0 \\
0 \\
b(t)
\end{array}\right) \text {, where } \quad \boldsymbol{B}\left(t^{\prime}\right)=\left(\begin{array}{ccc}
-(1 / \tau+i \Omega) & 0 & \frac{1}{2} i \omega_{\mathrm{R}} e^{-i k t^{\prime}} e^{i \phi} \\
0 & -(1 / \tau-i \Omega) & -\frac{1}{2} i \omega_{\mathrm{R}} e^{i k t^{\prime}} e^{-i \phi} \\
i \omega_{\mathrm{R}} e^{i k t^{\prime}} e^{-i \phi} & -i \omega_{\mathrm{R}} e^{-i k t^{\prime}} e^{i \phi} & -2 / \tau
\end{array}\right),
$$

with the initial conditions given at $t^{\prime}=t$ by $x_{1}(t, t), x_{2}(t, t)$, and $x_{3}(t, t)$. Here $b(t)$ is the inhomogeneous term, independent of $t^{\prime}$. The solution method we use is the same as the one in Refs. [2,15]. We begin by writing down the equations separately as

$$
\begin{aligned}
\frac{d x_{1}}{d t^{\prime}}+\left(\frac{1}{\tau}+i \Omega\right) x_{1} & =\frac{1}{2} i \omega_{\mathrm{R}} e^{-i k t^{\prime}} e^{i \phi} x_{3}, \quad \frac{d x_{2}}{d t^{\prime}}+\left(\frac{1}{\tau}-i \Omega\right) x_{2}=-\frac{1}{2} i \omega_{\mathrm{R}} e^{i k t^{\prime}} e^{-i \phi} x_{3}, \\
\frac{d x_{3}}{d t^{\prime}}+\frac{2}{\tau} x_{3} & =i \omega_{\mathrm{R}}\left(e^{i k t^{\prime}} e^{-i \phi} x_{1}-e^{-i k t^{\prime}} e^{i \phi} x_{2}\right) .
\end{aligned}
$$

Integrating $x_{1}\left(t, t^{\prime}\right)$ from $t^{\prime}=t$ to $t+\delta t$ and making a change of variables results in

$$
x_{1}(t, t+\delta t)=x_{1}(t, t) e^{-(1 / \tau+i \Omega) \delta t}+i \frac{\omega_{\mathrm{R}}}{2} e^{i \phi} e^{-(1 / \tau+i \Omega)(t+\delta t)} \int_{0}^{\delta t} d m e^{[1 / \tau+i(\Omega-k)](t+m)} x_{3}(t, t+m) .
$$

Likewise, for $x_{2}$ and $x_{3}$ we get

$$
\begin{aligned}
x_{2}(t, t+\delta t)= & x_{2}(t, t) e^{-(1 / \tau-i \Omega) \delta t}-i \frac{\omega_{\mathrm{R}}}{2} e^{-i \phi} e^{-(1 / \tau-i \Omega)(t+\delta t)} \int_{0}^{\delta t} d m e^{[1 / \tau-i(\Omega-k)](t+m)} x_{3}(t, t+m), \\
x_{3}(t, t+\delta t)= & x_{3}(t, t) e^{-\frac{2}{\tau} \delta t}+\frac{\tau}{2} b(t)\left(1-e^{-\frac{2}{\tau} \delta t}\right)+i \omega_{\mathrm{R}} e^{-i \phi} \int_{0}^{\delta t} d m e^{\frac{2}{\tau} m} e^{i k(t+m)} e^{-\frac{2}{\tau} \delta t} x_{1}(t, t+m) \\
& -i \omega_{\mathrm{R}} e^{i \phi} \int_{0}^{\delta t} d m e^{\frac{2}{\tau} m} e^{-i k(t+m)} e^{-\frac{2}{\tau} \delta t} x_{2}(t, t+m) .
\end{aligned}
$$

Substituting (A1) and (A2) in Eq. (A3) results in

$$
\begin{aligned}
x_{3}(t, t+\delta t)= & x_{3}(t, t) e^{-\frac{2}{\tau} \delta t}+\frac{\tau}{2} b(t)\left(1-e^{-\frac{2}{\tau} \delta t}\right)+i \omega_{\mathrm{R}} e^{-i \phi} x_{1}(t, t) e^{i k t} \frac{e^{-\left[\frac{1}{\tau}+i(\Omega-k)\right] \delta t}-e^{-\frac{2}{\tau} \delta t}}{\frac{1}{\tau}-i(\Omega-k)} \\
& -i \omega_{\mathrm{R}} e^{i \phi} x_{2}(t, t) e^{-i k t} \frac{e^{-\left[\frac{1}{\tau}-i(\Omega-k)\right] \delta t}-e^{-\frac{2}{\tau} \delta t}}{\frac{1}{\tau}+i(\Omega-k)}-\frac{\omega_{\mathrm{R}}^{2}}{2} \int_{0}^{\delta t} d m^{\prime} x_{3}\left(t, t+m^{\prime}\right) \frac{e^{-\left[\frac{1}{\tau}+i(\Omega-k)\right]\left(\delta t-m^{\prime}\right)}-e^{-\frac{2}{\tau}\left(\delta t-m^{\prime}\right)}}{\frac{1}{\tau}-i(\Omega-k)} \\
& -\frac{\omega_{\mathrm{R}}^{2}}{2} \int_{0}^{\delta t} d m^{\prime} x_{3}\left(t, t+m^{\prime}\right) \frac{e^{-\left[\frac{1}{\tau}-i(\Omega-k)\right]\left(\delta t-m^{\prime}\right)}-e^{-\frac{2}{\tau}\left(\delta t-m^{\prime}\right)}}{\frac{1}{\tau}+i(\Omega-k)}
\end{aligned}
$$

Once we take the Laplace transform of these equations with respect to the $\delta t$ variable, the convolution integrals simplify and we are left with

$$
\begin{aligned}
X_{3}(s)= & \frac{\left(s+\frac{2}{\tau}\right)\left[\left(s+\frac{1}{\tau}\right)^{2}+(\Omega-k)^{2}\right]}{\left(s+\frac{2}{\tau}\right)\left[\left(s+\frac{1}{\tau}\right)^{2}+(\Omega-k)^{2}\right]+\omega_{\mathrm{R}}^{2}\left(s+\frac{1}{\tau}\right)}\left\{x_{3}(t, t) \frac{1}{s+\frac{2}{\tau}}+\frac{\tau}{2} b(t)\left(\frac{1}{s}-\frac{1}{s+\frac{2}{\tau}}\right)\right. \\
& \left.+i \omega_{\mathrm{R}} e^{-i \phi} x_{1}(t, t) e^{i k t} \frac{1}{\left(s+\frac{2}{\tau}\right)\left[s+\frac{1}{\tau}+i(\Omega-k)\right]}-i \omega_{\mathrm{R}} e^{i \phi} x_{2}(t, t) e^{-i k t} \frac{1}{\left(s+\frac{2}{\tau}\right)\left[s+\frac{1}{\tau}-i(\Omega-k)\right]}\right\}
\end{aligned}
$$

Using (A1) and (A2) we get

$$
\begin{aligned}
& X_{1}(s)=x_{1}(t, t) \frac{1}{s+\frac{1}{\tau}+i \Omega}+i \frac{\omega_{\mathrm{R}}}{2} e^{i \phi} e^{-i k t} X_{3}(s+i k) \frac{1}{s+\frac{1}{\tau}+i \Omega}, \\
& X_{2}(s)=x_{2}(t, t) \frac{1}{s+\frac{1}{\tau}-i \Omega}-i \frac{\omega_{\mathrm{R}}}{2} e^{-i \phi} e^{i k t} X_{3}(s-i k) \frac{1}{s+\frac{1}{\tau}-i \Omega} .
\end{aligned}
$$

These results are the general solution to the Bloch equations expressed in the Laplace domain. 


\section{APPENDIX B: CORRELATION FUNCTION CALCULATIONS}

\section{Single time correlations}

The calculation of $\left\langle\sigma_{-}(t)\right\rangle,\left\langle\sigma_{+}(t)\right\rangle$, and $\left\langle\sigma_{z}(t)\right\rangle$ can be made by using the results from the previous section. The inhomogeneous term is $b(t)=-\frac{2}{\tau}$. We assume that the atom is initially in its ground state such that $x_{1}(0)=x_{2}(0)=0, x_{3}(0)=-1$. The Laplace transforms of the expectation values are

$$
\begin{aligned}
\mathcal{L}_{t}\left[e^{i k t}\left\langle\sigma_{-}(t)\right\rangle\right] & =-i \frac{\omega_{\mathrm{R}}}{2} e^{i \phi} \frac{\left(s+\frac{2}{\tau}\right)\left[s+\frac{1}{\tau}-i(\Omega-k)\right]}{s P(s)}, \\
\mathcal{L}_{t}\left[e^{-i k t}\left\langle\sigma_{+}(t)\right\rangle\right] & =i \frac{\omega_{\mathrm{R}}}{2} e^{-i \phi} \frac{\left(s+\frac{2}{\tau}\right)\left[s+\frac{1}{\tau}+i(\Omega-k)\right]}{s P(s)}, \\
\mathcal{L}_{t}\left[\left\langle\sigma_{z}(t)\right\rangle\right] & =\frac{-\left(s+\frac{2}{\tau}\right)\left[\left(s+\frac{1}{\tau}\right)^{2}+(\Omega-k)^{2}\right]}{s P(s)},
\end{aligned}
$$

where

$$
P(s)=\left(s+\frac{2}{\tau}\right)\left[\left(s+\frac{1}{\tau}\right)^{2}+(\Omega-k)^{2}\right]+\omega_{\mathrm{R}}^{2}\left(s+\frac{1}{\tau}\right) .
$$

The $t \rightarrow \infty$ limit of these quantities is also of interest. We get

$$
\begin{aligned}
\lim _{t \rightarrow \infty}\left\langle\sigma_{-}(t)\right\rangle & =\frac{-\frac{i}{2} R(1-i D)}{1+D^{2}+\frac{1}{2} R^{2}} e^{-i k t+i \phi}, \quad \lim _{t \rightarrow \infty}\left\langle\sigma_{+}(t)\right\rangle=\frac{\frac{i}{2} R(1+i D)}{1+D^{2}+\frac{1}{2} R^{2}} e^{i k t-i \phi}, \\
\lim _{t \rightarrow \infty} \frac{\left\langle\sigma_{z}(t)\right\rangle+1}{2} & =\lim _{t \rightarrow \infty}\left\langle\sigma_{+}(t) \sigma_{-}(t)\right\rangle=\frac{\frac{1}{4} R^{2}}{1+D^{2}+\frac{1}{2} R^{2}} .
\end{aligned}
$$

Here $D \equiv(\Omega-k) \tau$ stands for the normalized detuning frequency and $R \equiv \omega_{\mathrm{R}} \tau$ for the normalized Rabi frequency.

\section{Double time correlations of two operators}

To calculate $\left\langle\sigma_{+}(t) \sigma_{-}\left(t^{\prime}\right)\right\rangle,\left\langle\sigma_{+}(t) \sigma_{+}\left(t^{\prime}\right)\right\rangle$, and $\left\langle\sigma_{+}(t) \sigma_{z}\left(t^{\prime}\right)\right\rangle$ we use initial values at $t^{\prime}=t$ under steady-state conditions when $t \rightarrow \infty$ such that $\left\langle\sigma_{+}(t) \sigma_{-}(t)\right\rangle$ is given by Eq. (B6), $\left\langle\sigma_{+}(t) \sigma_{+}(t)\right\rangle=0$ and $\left\langle\sigma_{+}(t) \sigma_{z}(t)\right\rangle=-\left\langle\sigma_{+}(t)\right\rangle$ is given by Eq. (B5). The inhomogeneous term is $b(t)=-\frac{2}{\tau}\left\langle\sigma_{+}(t)\right\rangle$. After some algebra we get

$$
\begin{aligned}
\mathcal{L}_{\delta t}\left[e^{i k \delta t}\left\langle\sigma_{+}(t) \sigma_{-}(t+\delta t)\right\rangle\right] & =\frac{\frac{1}{4} R^{2}}{1+D^{2}+\frac{1}{2} R^{2}} \frac{P(s)-\frac{1}{2} \omega_{\mathrm{R}}^{2}\left(s+\frac{2}{\tau}\right)}{s P(s)}, \\
\mathcal{L}_{\delta t}\left[e^{-i k \delta t}\left\langle\sigma_{+}(t) \sigma_{+}(t+\delta t)\right\rangle\right] & =\frac{-\frac{1}{4} R^{2} e^{-2 i \phi+2 i k t}}{1+D^{2}+\frac{1}{2} R^{2}} \frac{\frac{P(s)-\frac{1}{2} \omega_{\mathrm{R}}^{2}\left(s+\frac{2}{\tau}\right)}{s P(s)}\left[s+\frac{1}{\tau}+i(\Omega-k)\right]-1}{s+\frac{1}{\tau}-i(\Omega-k)} \\
\mathcal{L}_{\delta t}\left[\left\langle\sigma_{+}(t) \sigma_{z}(t+\delta t)\right\rangle\right] & =\frac{-\frac{i}{2} R \tau e^{-i \phi+i k t}}{1+D^{2}+\frac{1}{2} R^{2}}\left\{\frac{P(s)-\frac{1}{2} \omega_{\mathrm{R}}^{2}\left(s+\frac{2}{\tau}\right)}{s P(s)}\left[s+\frac{1}{\tau}+i(\Omega-k)\right]-1\right\} .
\end{aligned}
$$

Note that the Laplace transforms are taken with respect to $\delta t$.

\section{Double time correlations of three operators}

We multiply input-output equations (3) evaluated at time $t^{\prime}$ by $\sigma_{+}(t)$ from the left and $\sigma_{-}(t)$ from the right and take the expectation values to arrive at the double time correlations of three operators. The initial values are given by

$$
\left\langle\sigma_{+}(t) \sigma_{-}(t) \sigma_{-}(t)\right\rangle=\left\langle\sigma_{+}(t) \sigma_{+}(t) \sigma_{-}(t)\right\rangle=0, \quad\left\langle\sigma_{+}(t) \sigma_{z}(t) \sigma_{-}(t)\right\rangle=-\left\langle\sigma_{+}(t) \sigma_{-}(t)\right\rangle=-\frac{\left\langle\sigma_{z}(t)+1\right\rangle}{2},
$$

and the inhomogeneous term is

$$
b(t)=-\frac{2}{\tau} \frac{\left\langle\sigma_{z}(t)+1\right\rangle}{2} .
$$


The expectation value of $\sigma_{z}(t)$ is at its steady-state value given by Eq. (B6). If we compare the initial values and the inhomogeneous term to the case of single time correlations, we see that they are exactly the same except for the scaling term $\left\langle\sigma_{z}(t)+1\right\rangle / 2$ which is given by Eq. (B6). Thus the results are just rescaled versions of the single time correlation ones and are given by

$$
\begin{aligned}
\mathcal{L}_{\delta t}\left[e^{i k \delta t}\left\langle\sigma_{+}(t) \sigma_{-}(t+\delta t) \sigma_{-}(t)\right\rangle\right] & =(\mathrm{B} 1) \times(\mathrm{B} 6), \quad \mathcal{L}_{\delta t}\left[e^{-i k \delta t}\left\langle\sigma_{+}(t) \sigma_{+}(t+\delta t) \sigma_{-}(t)\right\rangle\right]=(\mathrm{B} 2) \times(\mathrm{B} 6), \\
\mathcal{L}_{\delta t}\left[\left\langle\sigma_{+}(t) \sigma_{z}(t+\delta t) \sigma_{-}(t)\right\rangle\right] & =(\mathrm{B} 3) \times(\mathrm{B} 6),
\end{aligned}
$$

where the Laplace transforms are taken with respect to $\delta t$.

\section{APPENDIX C: SHORT NOTE ON NUMERICS}

The differential equations that we analyzed so far can be transformed into time-independent forms by the substitution

$$
\tilde{\sigma}_{-}=e^{i k t} \sigma_{-}, \quad \tilde{\sigma}_{+}=e^{-i k t} \sigma_{+}, \quad \text { and } \quad \tilde{\sigma}_{z}=\sigma_{z}
$$

where $k$ is the frequency of the incoming photons. For instance, the single time expectation values of $\tilde{\sigma}_{-}, \tilde{\sigma}_{+}$, and $\tilde{\sigma}_{z}$ can be written as

$$
\frac{d}{d t}\left(\begin{array}{c}
\left\langle\tilde{\sigma}_{-}\right\rangle \\
\left\langle\tilde{\sigma}_{+}\right\rangle \\
\left\langle\tilde{\sigma}_{z}\right\rangle
\end{array}\right)=\boldsymbol{M}\left(\begin{array}{c}
\left\langle\tilde{\sigma}_{-}\right\rangle \\
\left\langle\tilde{\sigma}_{+}\right\rangle \\
\left\langle\tilde{\sigma}_{z}\right\rangle
\end{array}\right)+\left(\begin{array}{c}
0 \\
0 \\
-\frac{2}{\tau}
\end{array}\right),
$$

where the matrix $\boldsymbol{M}$ is given by

$$
\boldsymbol{M}=\left(\begin{array}{lcc}
-\left(\frac{1}{\tau}+i \tilde{\Omega}\right) & 0 & i \frac{\omega_{\mathrm{R}}}{2} e^{i \phi} \\
0 & -\left(\frac{1}{\tau}-i \tilde{\Omega}\right) & -i \frac{\omega_{\mathrm{R}}}{2} e^{-i \phi} \\
i \omega_{\mathrm{R}} e^{-i \phi} & -i \omega_{\mathrm{R}} e^{i \phi} & -\frac{2}{\tau}
\end{array}\right),
$$

and $\tilde{\Omega} \equiv \Omega-k$. Other expectation values have the same form as well. This is a much more convenient formulation for purely numerical studies with which we verified the analytical results reported in the previous Appendices.

[1] B. R. Mollow, Phys. Rev. 188, 1969 (1969).

[2] H. J. Kimble and L. Mandel, Phys. Rev. A 13, 2123 (1976).

[3] J. L. O’Brien, A. Furusawa, and J. Vuckovic, Nat. Photon. 3, 687 (2009).

[4] O. Benson, Nature (London) 480, 193 (2011).

[5] J. Q. You and F. Nori, Nature (London) 474, 589 (2011).

[6] O. Astafiev, A. M. Zagoskin, A. A. Abdumalikov Jr., Yu. A. Pashkin, T. Yamamoto, K. Inomata, Y. Nakamura, and J. S. Tsai, Science 327, 840 (2010).

[7] D. Bozyigit, C. Lang, L. Steffen, J. M. Fink, C. Eichler, M. Baur, R. Bianchetti, P. J. Leek, S. Filipp, M. P. da Silva, A. Blais, and A. Wallraff, Nat. Phys. 7, 154 (2011).

[8] M. P. da Silva, D. Bozyigit, A. Wallraff, and A. Blais, Phys. Rev. A 82, 043804 (2010).

[9] A. A. Abdumalikov, O. V. Astafiev, Y. A. Pashkin, Y. Nakamura, and J. S. Tsai, Phys. Rev. Lett. 107, 043604 (2011).

[10] A. A. Abdumalikov, O. Astafiev, A. M. Zagoskin, Y. A. Pashkin, Y. Nakamura, and J. S. Tsai, Phys. Rev. Lett. 104, 193601 (2010).

[11] I.-C. Hoi, C. M. Wilson, G. Johansson, T. Palomaki, B. Peropadre, and P. Delsing, Phys. Rev. Lett. 107, 073601 (2011).

[12] M. Florescu and S. John, Phys. Rev. A 69, 053810 (2004).

[13] M. Davanço and K. Srinivasan, Opt. Lett. 34, 2542 (2009).

[14] M. Davanço, M. T. Rakher, W. Wegscheider, D. Schuh, A. Badolato, and K. Srinivasan, Appl. Phys. Lett. 99, 121101 (2011).
[15] L. Mandel and E. Wolf, Optical Coherence and Quantum Optics (Cambridge University Press, Cambridge, England, 1995), Sec. 15.6.

[16] H. Carmichael, Statistical Methods in Quantum Optics 1: Master Equations and Fokker-Planck Equations (Springer, New York, 2002), Sec. 2.3.

[17] M. O. Scully and M. S. Zubairy, Quantum Optics (Cambridge University Press, Cambridge, England, 1997), Chap. 10.

[18] D. Walls and G. J. Milburn, Quantum Optics, 2nd ed. (Springer, New York, 2008), Sec. 10.5.

[19] M. Hennrich, A. Kuhn, and G. Rempe, Phys. Rev. Lett. 94, 053604 (2005).

[20] F. Le Kien and K. Hakuta, Phys. Rev. A 77, 033826 (2008).

[21] L. Jin, J. Evers, and M. Macovei, Phys. Rev. A 84, 043812 (2011).

[22] J. T. Shen and S. Fan, Phys. Rev. Lett. 98, 153003 (2007); Phys. Rev. A 76, 062709 (2007).

[23] C. W. Gardiner and M. J. Collett, Phys. Rev. A 31, 3761 (1985).

[24] S. Fan, Ş. E. Kocabaş, and J.-T. Shen, Phys. Rev. A 82, 063821 (2010).

[25] This commutator identity for $t^{\prime}>t$ is proved using causality in Ref. [23]. For $t^{\prime}=t$, the identity can be directly proved using the definition of the operators, as shown in the Appendix of E. Rephaeli, Ş. E. Kocabaş, and S. Fan, Phys. Rev. A 84, 063832 (2011). 
[26] S. M. Barnett and P. M. Radmore, Methods in Theoretical Quantum Optics (Oxford University Press, New York, 1997), Sec. 3.6.

[27] See Supplemental Material at http://link.aps.org/supplemental/ 10.1103/PhysRevA.85.023817 for the MATHEMATICA source code used in the derivations.

[28] D. Chang, A. Sørensen, E. Demler, and M. Lukin, Nature Physics 3, 807 (2007).

[29] H. Zheng, D. J. Gauthier, and H. U. Baranger, Phys. Rev. A 82, 063816 (2010).
[30] I.-C. Hoi, T. Palomaki, G. Johansson, J. Lindkvist, P. Delsing, and C. M. Wilson, e-print arXiv:1201.2269.

[31] D. I. Schuster, A. Wallraff, A. Blais, L. Frunzio, R.-S. Huang, J. Majer, S. M. Girvin, and R. J. Schoelkopf, Phys. Rev. Lett. 94, 123602 (2005).

[32] A. Fragner, M. Göppl, J. M. Fink, M. Baur, R. Bianchetti, P. J. Leek, A. Blais, and A. Wallraff, Science 322, 1357 (2008).

[33] M. Koch, C. Sames, M. Balbach, H. Chibani, A. Kubanek, K. Murr, T. Wilk, and G. Rempe, Phys. Rev. Lett. 107, 023601 (2011). 